\title{
VARIABILIDADE ESPACIAL DA SALINIDADE DE UM SOLO ALUVIAL NO SEMI-ÁRIDO PARAIBANO ${ }^{1}$
}

\author{
Lázaro Costa de Souza², José Elenildo Queiroz ${ }^{3}$ \& Hans Raj Gheyi ${ }^{4}$
}

\begin{abstract}
RESUMO
O objetivo deste trabalho foi estudar a variabilidade espacial do $\mathrm{pH}$ da pasta de saturação (pHps), condutividade elétrica do extrato de saturação (CEes) e porcentagem de sódio trocável (PST) de um solo aluvial afetado por sais. Foram coletadas amostras de solo nas camadas 0-20, $20-40$ e 40-60 cm, numa área do Projeto de Irrigação Capoeira, localizado em São José do Bonfim, Estado da Paraíba, utilizando-se um esquema sistemático de amostragem numa malha regular de $10 \times 15 \mathrm{~m}$. Os dados foram analisados através da estatística descritiva e geoestatística. Verificou-se baixa variabilidade para o pHps $(\mathrm{CV}<12 \%)$ e elevada para a CEes e PST $(\mathrm{CV}>60 \%)$. Modelos esférico e gaussiano foram ajustados aos semivariogramas das variáveis que apresentaram estrutura de dependência espacial, tendo-se obtido alcances variando de 20 a $40 \mathrm{~m}$. Os mapas de isolinhas da combinação da CEes e PST permitiram visualizar o padrão de variabilidade da salinidade e sodicidade, constituindo-se em uma ferramenta para a definição de estratégias de manejo edáfico e recuperação da área afetada.
\end{abstract}

Palavras-chave: salinização, porcentagem de sódio trocável, geoestatística

\section{SPATIAL VARIABILITY OF SOIL SALINITY IN AN ALLUVIAL SOIL OF THE SEMI-ARID REGION OF PARAIIBA STATE}

\begin{abstract}
The objective of this work was to study the spatial variability of $\mathrm{pH}$ of saturation paste (pHsp), the electrical conductivity of saturation extract (ECe) and the exchangeable sodium percentage (ESP) in an alluvial salt affected soil. Soil samples were collected in 0 - 20, 20 - 40 and $40-60 \mathrm{~cm}$ depths, in an area of the Irrigation Project of Capoeira, located in São José do Bonfim - Paraíba (Brazil), following a systematic scheme of sampling in a $10 \times 15 \mathrm{~m}$ mesh. The data were analyzed through the descriptive statistics and geostatistics. Low variability was observed for the pHsp $(\mathrm{CV}<12 \%)$ in constrast to ECe and $\operatorname{ESP}(\mathrm{CV}>60 \%)$. Spherical and gaussian models were adjusted to the experimental semivariograms of the variables that presented a spatial dependence structure. Structures of spatial dependence, with range varying from 20 to $40 \mathrm{~m}$ were observed. The maps of isohypsas of the combination of ECe and ESP allowed the visualization of the pattern of the salinity and sodicity variability, constituting a tool for the definition of soil management and reclamation of the affected area.
\end{abstract}

Key words: salinization, exchangeable sodium percentage, geostatistics

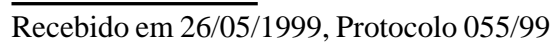

${ }^{1}$ Parte de dissertação de Mestrado do primeiro autor, apresentada ao Curso de Pós-Graduação em Engenharia Agrícola da Universidade Federal da Paraíba, Campina Grande, PB, Campus II

${ }^{2}$ Engenheiro Agrônomo, Mestre em Engenharia Agrícola, Pesquisador da EMEPA - PB

${ }^{3}$ Doutor, Prof. Adjunto do Depto. de Eng. Florestal da UFPB, Campus VII, CP 64, CEP 58700 - 970 , Patos, PB. E-mail: elenildo@cstr.ufpb.br

${ }^{4}$ Doutor, Prof. Titular do Depto. de Eng. Agrícola da UFPB, Campus II. Av. Aprígio Veloso 882, Bodocongó, CEP 58109 - 970 , Campina Grande, PB. Fone: (0xx83) 310 1056, Fax: (0xx83) 310 1185. E-mail:hans@ deag.ufpb.br 


\section{INTRODUÇÃO}

A salinidade do solo constitui um sério problema nas áreas irrigadas, principalmente quando sua intensidade interfere no desenvolvimento das culturas, reduzindo a produção e a produtividade das plantas, a níveis antieconômicos (Oliveira, 1997). Este problema é mais freqüente em regiões tropicais de clima quente e seco, caracterizado por elevadas taxas de evapotranspiração e baixos índices pluviométricos, a exemplo do semi-árido do Nordeste brasileiro, onde se concentram, atualmente, as terras mais intensamente cultivadas; com o uso da irrigação, o risco de tornar os solos salinizados ou alcalinizados é mais sério ainda. FAO/UNESCO (1973) destaca que os aspectos edafoclimáticos, presença de lençóis freáticos elevados, irrigação e drenagem inadequadas, em muito contribuem para o processo. O conjunto desses fatores e a ação do homem, sob diferentes níveis, imprimem uma grande variabilidade aos solos (Albuquerque et al., 1996).

Os solos podem apresentar diferenças marcantes em suas propriedades físico-químicas, até mesmo a curtas distâncias, tanto vertical como horizontalmente (Beckett \& Webster, 1971) o que dificulta o manejo de solo e água, principalmente nos solos aluviais que, apesar dos problemas que apresentam, são bastante usados nas atividades agrícolas. Isto demonstra a necessidade de pesquisas que proporcionem melhor avaliação de sua variabilidade espacial, visando à adoção de técnicas adequadas de manejo agrícola.

Este trabalho teve como objetivo estudar a variabilidade espacial das variáveis indispensáveis à caracterização da salinidade tais como $\mathrm{pH}$ da pasta de saturação do solo (pHps), condutividade elétrica do extrato de saturação (CEes) e porcentagem de sódio trocável (PST), em um solo de origem aluvial no semi-árido paraibano, utilizando-se de técnicas estatísticas descritivas e geoestatísticas para identificação dos padrões de variabilidade e dependência espacial das variáveis estudadas, visando ao mapeamento da salinidade e sodicidade e fornecimento de subsídios para um manejo mais adequado do solo na área analisada.

\section{MATERIAL E MÉTODOS}

O trabalho foi realizado em uma área de solo aluvial do Projeto de Irrigação Capoeira, localizado no município de São José do Bonfim, Estado da Paraíba, situado entre as coordenadas geográficas $7^{\circ} 00^{\prime}$ e $7^{\circ} 30^{\prime}$ 'S e $37^{\circ} 10^{\prime}$ e $37^{\circ} 30^{\prime}$ W. Pela classificação de Köppen, o clima da região é do tipo semi-árido quente e seco (Bsh), com precipitação média de $400 \mathrm{~mm}$ e uma estação seca bem definida; as temperaturas médias nunca são inferiores a $24^{\circ} \mathrm{C}$ com amplitude de $5^{\circ} \mathrm{C}$ (Brasil/Sudene, 1972). Os solos da maior parte do projeto são constituídos de várias unidades do tipo aluvial (Paraíba, 1989).

Foi utilizado um sistema de amostragem sistemática em uma malha regular de $10 \times 15 \mathrm{~m}$ (120 pontos amostrais) coletando-se amostras nas camadas 0 - 20, 20 - 40 e $40-60 \mathrm{~cm}$, totalizando 360 amostras. Foram analisadas as variáveis: pHps, CEes, cátions solúveis do extrato de saturação, seguindo-se procedimentos descritos por Richards (1954) e cátions totais, conforme metodologia da Embrapa (1997); os cátions trocáveis foram obtidos pela diferença entre totais e solúveis. A capacidade de troca de cátions (CTC) foi obtida através da soma dos cátions trocáveis e utilizada no cálculo da PST, obtida da relação Na trocável x 100/CTC.
Inicialmente, foi realizada uma análise descritiva, com o objetivo de se observar o comportamento geral dos dados, através do cálculo das medidas estatísticas de posição e dispersão (magnitude de variabilidade, presença de valores atípicos, tendência central etc.) e a aderência à distribuição normal. Os dados discrepantes foram eliminados com base no critério de Hoaglin et al. (1983) que consideram discrepantes aqueles dados abaixo do limite inferior ( $\mathrm{Li}$ ) ou acima do limite superior (Ls) respectivamente, estimados por: $\mathrm{Li}=\mathrm{Qi}-1,5 \mathrm{Ai}$ e $\mathrm{Ls}=\mathrm{Qs}+1,5 \mathrm{Ai}$, sendo Qi e Qs os quartis inferior e superior, respectivamente, e Ai a amplitude interquartílica. A estrutura de dependência espacial foi estudada por meio da geoestatística, através de semivariogramas construídos com base nas semivariâncias estimadas por:

$$
\gamma *(\mathrm{~h})=\frac{1}{2 \mathrm{~N}(\mathrm{~h})} \sum_{\mathrm{i}=1}^{\mathrm{N}(\mathrm{h})}\left[\mathrm{Z}\left(\mathrm{x}_{\mathrm{i}}\right)-\mathrm{Z}\left(\mathrm{x}_{\mathrm{i}}+\mathrm{h}\right)\right]^{2}
$$

em que $\gamma^{*}$ é o valor da semivariância estimada a partir dos dados experimentais; $N(h)$ é o número de pares de observações $\mathrm{Z}\left(\mathrm{x}_{\mathrm{i}}\right)$ e $\mathrm{Z}\left(\mathrm{x}_{\mathrm{i}}+\mathrm{h}\right)$ separadas por uma distância $\mathrm{h}$ (Guerra, 1988; Carvalho, 1996).

Aos semivariogramas experimentais que apresentaram estrutura de dependência espacial, ajustaram-se modelos esférico e gaussiano, respectivamente, conforme as Eq. (2) e (3):

$$
\begin{gathered}
\gamma(\mathrm{h})=\mathrm{Co}+\mathrm{Cl}\left[\frac{1}{2}\left(\frac{\mathrm{h}}{\mathrm{a}}\right)-\frac{3}{2}\left(\frac{\mathrm{h}}{\mathrm{a}}\right)^{3}\right] \text { para } 0<\mathrm{h}<\mathrm{a} \\
\gamma(\mathrm{h})=\mathrm{Co}+\mathrm{Cl} ; \quad \mathrm{h} \geq \mathrm{a} \\
\gamma^{*}(\mathrm{~h})=\mathrm{Co}+\mathrm{Cl}\left\{1-\exp \left(\frac{-3 \mathrm{~h}^{2}}{\mathrm{a}^{2}}\right)\right\} \text { para } \mathrm{h} \geq 0
\end{gathered}
$$

onde $\mathrm{Co}, \mathrm{C} 1, \mathrm{Co}+\mathrm{C} 1$ e a são denominados efeito pepita, variância espacial, patamar e alcance da dependência espacial, respectivamente.

Para fins de caracterização da salinidade na área estudada, foram confeccionados mapas de isolinhas da combinação dos dados da CEes e PST - obtidos de uma malha retangular de $70 \times 210 \mathrm{~m}$, com 15 linhas e 43 colunas, espaçadas em $5 \mathrm{~m}$ entre linhas e colunas, totalizando 645 pontos.

\section{RESULTADOS E DISCUSSÃO}

Na Tabela 1 observam-se as medidas estatísticas das variáveis pHps, CEes e PST referentes às profundidades 0 - 20, 20 - $40 \mathrm{e}$ $40-60 \mathrm{~cm}$, respectivamente. Observa-se, também, que os valores das médias e medianas da variável pHps são aproximadamente iguais em todas as profundidades estudadas, enquanto para a CEes e PST verificam-se elevadas diferenças entre essas medidas. A proximidade desses valores para o pHps é um indicativo de possíveis ajustes dos dados desta variável à distribuição normal. A aplicação do teste de Kolmogorov-Smirnov (KS) a 1\% de probabilidade (Costa Neto, 1997) confirmou esta hipótese, indicando que os dados de pHps podem ser considerados provenientes de uma população com distribuição normal. A análise conjunta dos valores das médias e medianas, coeficientes de assimetria e curtose e o uso do teste KS, evidenciam a não normalidade dos dados de CEes e PST nas profundidades analisadas. 
Tabela 1. Medidas estatísticas das variáveis $\mathrm{pH}$ da pasta saturada (pHps), condutividade elétrica do extrato de saturação (CEes) e percentagem do sódio trocável (PST) referentes às profundidades 0-20; 20-40 e 40-60 cm de solo do Projeto Capoeira

\begin{tabular}{|c|c|c|c|}
\hline Medida Estatística & $\mathrm{pHps}$ & CEes $\left(\mathrm{dS} \mathrm{m} \mathrm{m}^{-1}\right.$ a $\left.25^{\circ} \mathrm{C}\right) \mathrm{F}$ & PST \\
\hline \multicolumn{4}{|l|}{ Profundidade $(0-20 \mathrm{~cm})$} \\
\hline Média & 6,33 & 2,71 & 4,28 \\
\hline Mediana & 6,40 & 0,54 & 1,15 \\
\hline Coef. de variação (CV) & 8,25 & 173,15 & 172,61 \\
\hline Coef. de assimetria & 1,45 & 2,76 & 2,67 \\
\hline Coef. de curtose & 10,68 & 8,98 & 7,11 \\
\hline Amplitude total (At) & 4,32 & 27,98 & 34,81 \\
\hline $\mathrm{D}(\mathrm{KS}) *$ & $0,13 * *$ & 0,31 & 0,31 \\
\hline \multicolumn{4}{|l|}{ Profundidade $(20-40 \mathrm{~cm})$} \\
\hline Média & 6,46 & 2,96 & 4,96 \\
\hline Mediana & 6,40 & 0,55 & 1,49 \\
\hline Coef. de variação (CV) & 10,34 & 155,20 & 169,68 \\
\hline Coef. de assimetria & 1,25 & 2,27 & 2,37 \\
\hline Coef. de curtose & 4,40 & 5,18 & 5,27 \\
\hline Amplitude total (At) & 4,57 & 22,98 & 42,86 \\
\hline $\mathrm{D}(\mathrm{KS}) *$ & $0,07 * *$ & 0,27 & 0,34 \\
\hline \multicolumn{4}{|l|}{ Profundidade $(40-60 \mathrm{~cm})$} \\
\hline Média & 6,58 & 3,28 & 6,82 \\
\hline Mediana & 6,45 & 1,08 & 1,72 \\
\hline Coef. de variação (CV) & 10,90 & 135,84 & 157,95 \\
\hline Coef. de assimetria & 1,07 & 1,92 & 1,91 \\
\hline Coef. de curtose & 2,08 & 3,86 & 2,70 \\
\hline Amplitude total (At) & 4,16 & 23,29 & 45,95 \\
\hline $\mathrm{D}(\mathrm{KS})^{*}$ & $0,11 * *$ & 0,23 & 0,35 \\
\hline
\end{tabular}

Com base na classificação de Warrick \& Nielsen (1980) verifica-se baixa variação (CV de 8,25 a 10,91\%) para o pHps, mas elevada para a CEes (CV entre 135,85 e 173,15\%) e PST (CV de 157,95 a $172,61 \%$ ) em todas as profundidades estudadas (Tabela 1) atribuindo caráter heterogêneo ao solo da área, o que é próprio de solos aluvionais, concordando com Oliveira (1998). Esta heterogeneidade pode ser atribuída aos processos de formação inerentes a esses solos, que ocorre pela acumulação e distribuição desuniformes de sucessivos depósitos de materiais oriundos de outros lugares, proporcionados pela posição topográfica que ocupam, além do manejo a que são submetidos, entre outros. A baixa variação observada para o pHps talvez se deva à reposição de cargas hidrogeniônicas fornecidas pela matéria orgânica, propiciando certa estabilidade do $\mathrm{pHps}$ no solo estudado. Observa-se, ainda, na Tabela 1, que a menor variação evidenciada pela amplitude total (At) é da variável $\mathrm{pHps}$. Em ordem crescente de valores, a amplitude total de variação observada em todas as profundidades é a seguinte: $\mathrm{pH}<\mathrm{CEes}$ $<$ PST.

Para fins de análise geoestatística, a área foi dividida em duas sub-áreas: afetada por salinidade (A) e normal (N). As Figuras 1,2 e 3 e a Tabela 2 representam os semivariogramas experimentais das variáveis analisadas e os modelos ajustados para os casos que apresentaram estrutura de dependência espacial.

$\mathrm{Na}$ análise de semivariogramas, o efeito pepita $(\mathrm{Co})$ representa a descontinuidade do semivariograma atribuída a erros de amostragens ou variações locais, não explicados pela variabilidade dos dados para distâncias amostradas.
A. Área afetada SED
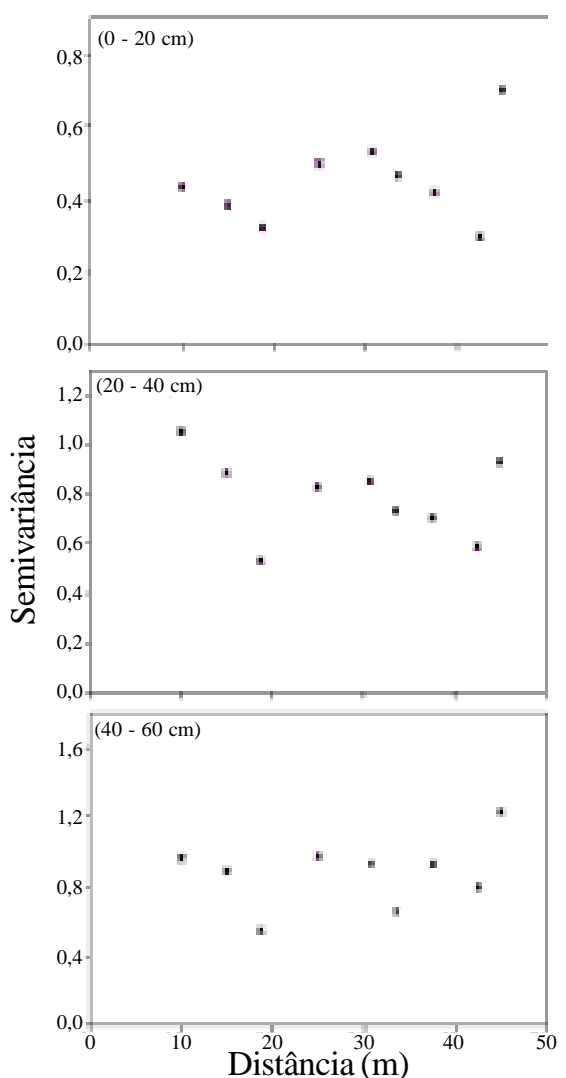

B. Área afetada CED
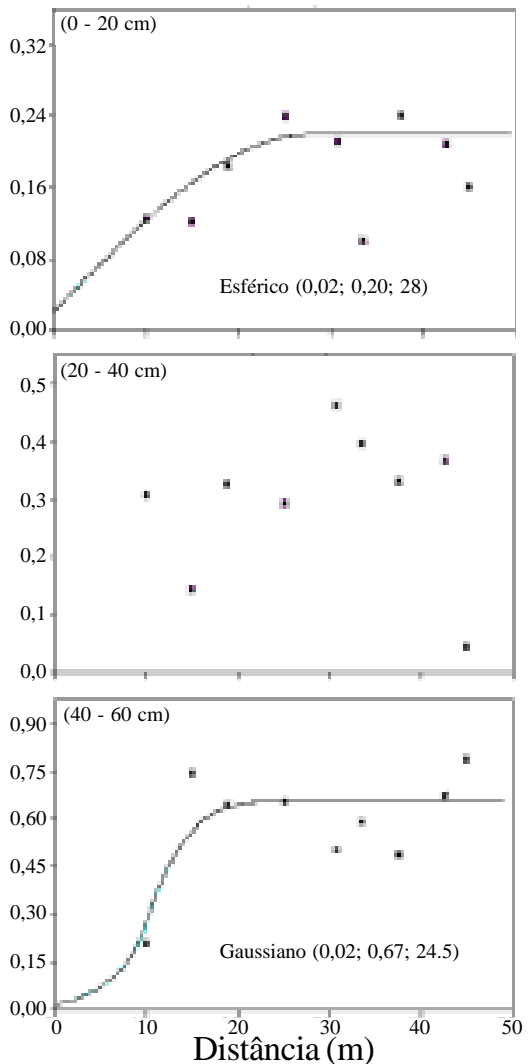

C. Área normal
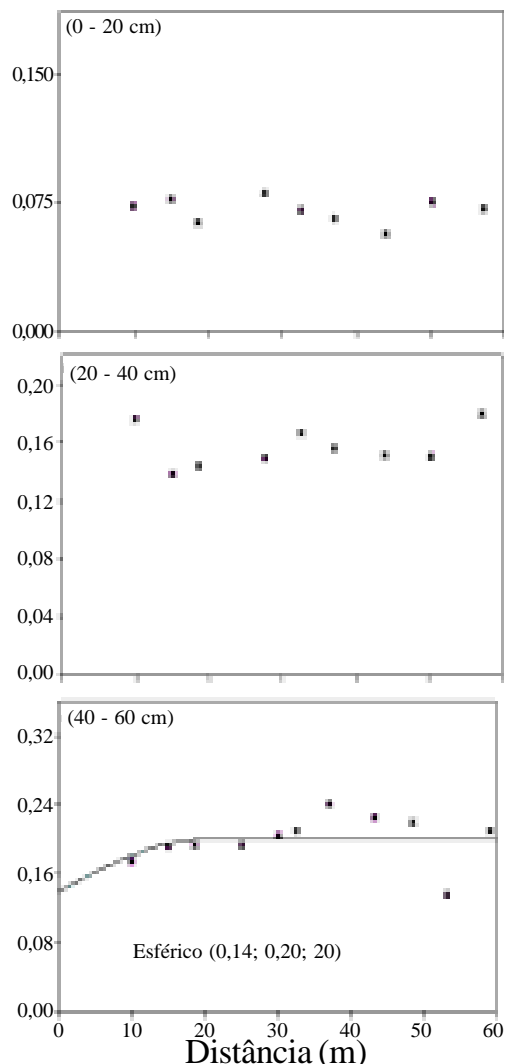

Figura 1. Semivariogramas experimentais e modelos ajustados para o pHps nas profundidades 0 - 20, 20 - 40 e 40 - $60 \mathrm{~cm}$ : (A) área afetada sem eliminação de dados discrepantes (SED); (B) área afetada com eliminação de dados discrepantes (CED) e (C) área normal 


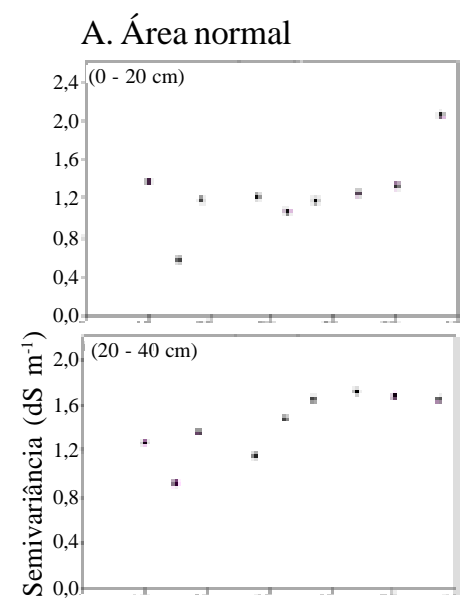

\section{B. Área afetada}
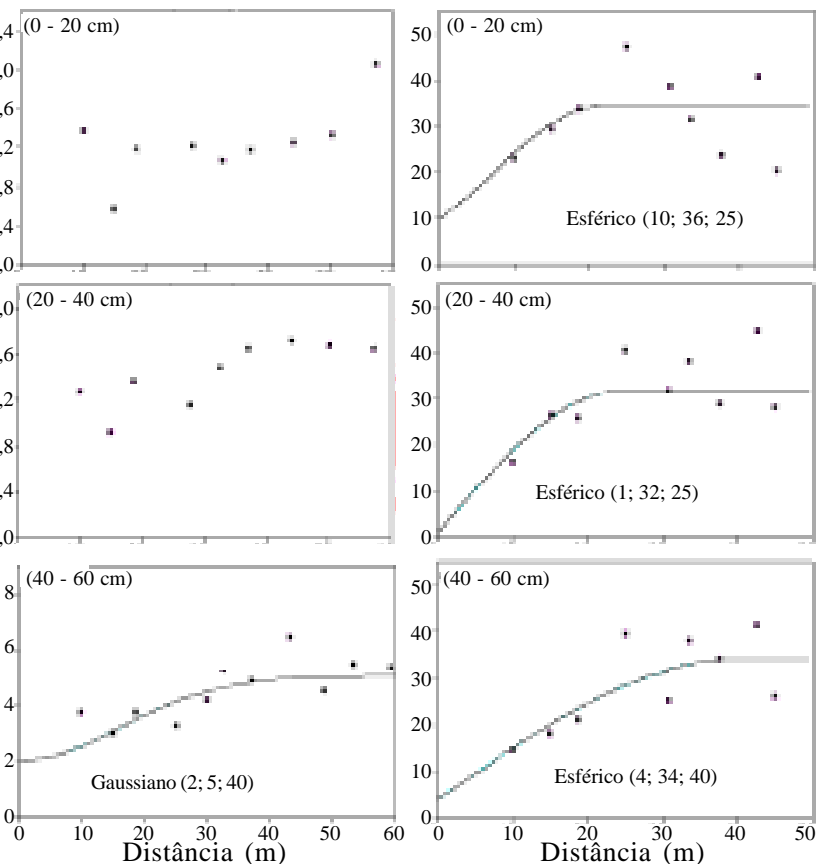

Figura 2. Semivariogramas experimentais e modelos ajustados da variável CEes para as áreas normal (A) e afetada (B) nas camadas 0 - 20, 20 - 40 e 40 - $60 \mathrm{~cm}$
A. Área normal

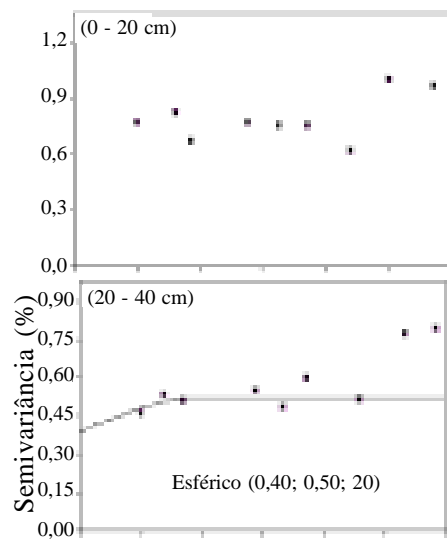

B. Área afetada
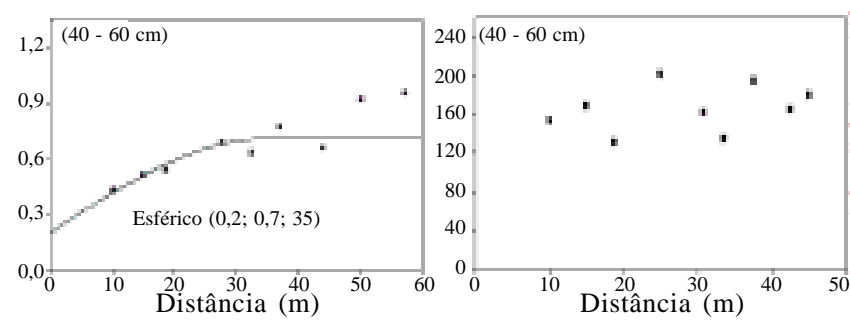

Figura 3. Semivariogramas experimentais e modelos ajustados da variável PST para as áreas normal (A) e afetada (B) nas camadas 0 - 20, 20 - 40 e $40-60 \mathrm{~cm}$

Tabela 2. Estimativas dos parâmetros do modelo teórico ajustado aos semivariogramas experimentais e a relação efeito pepita/patamar (expressa em porcentagem) para o pHps (sem e com a eliminação de dados discrepantes); CEes e PST, para as sub-áreas afetada e normal, nas camadas 0 - 20, 20 - 40 e 40 - $60 \mathrm{~cm}$

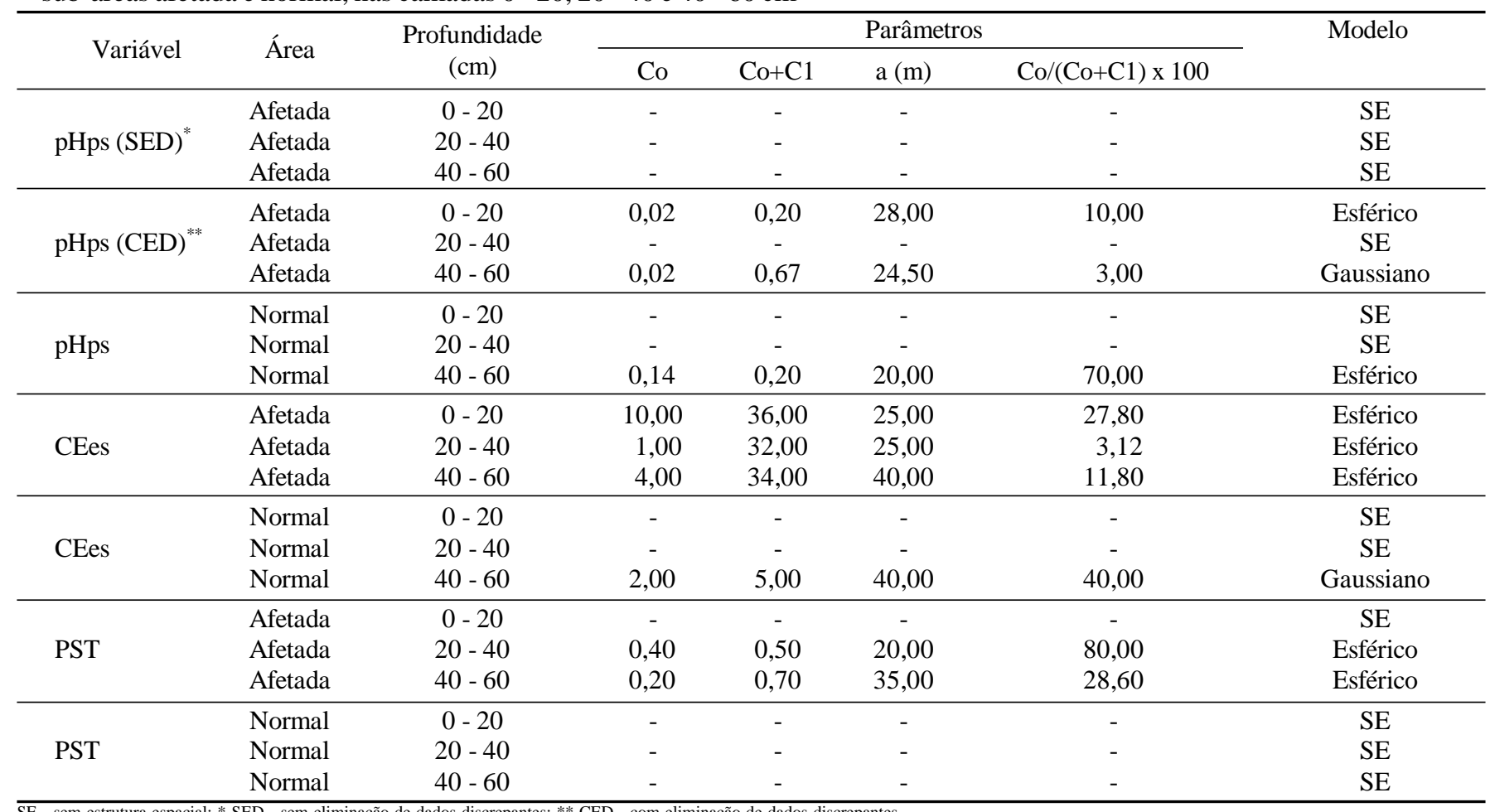

A classificação proposta por Cambardella et al. (1994) que consideram o efeito pepita como uma porcentagem do patamar, permite comparar o grau de dependência espacial de variáveis estudadas. Segundo esta classificação, semivariogramas com efeito pepita $<25 \%$, entre 25 e $75 \%$ e $>75 \%$, são considerados de forte, moderada e fraca dependência espacial, respectivamente. Na Tabela 2 observa-se que o pHps, após a eliminação de dados discrepantes, apresenta forte dependência espacial nas camadas $0-20$ e 40 - 60 cm na sub-área afetada e que, na sub-área normal, verifica-se moderada dependência espacial apenas na camada $40-60 \mathrm{~cm}$; nos demais casos, esta variável não apresenta estrutura de dependência espacial, o que concorda, em parte, com Carvalho (1996). 
Para a CEes na sub-área normal apenas na camada $40-60 \mathrm{~cm}$, constata-se moderada dependência espacial, enquanto as camadas $0-20$ e $20-40 \mathrm{~cm}$ não apresentam estrutura de dependência; na sub-área afetada esta variável apresenta moderada dependência espacial na camada $0-20 \mathrm{~cm}$ e forte dependência nas camadas 20 - 40 e $40-60 \mathrm{~cm}$, respectivamente (Figura $2 \mathrm{e}$ Tabela 2). Para a PST, devido ao elevado efeito pepita para a camada 20 - $40 \mathrm{~cm}$ na sub-área normal, observa-se fraca dependência espacial, enquanto na profundidade $40-60 \mathrm{~cm}$ a dependência espacial apresenta-se moderada; nos demais casos constata-se, através da Tabela 2 , que esta variável não apresenta estrutura de dependência espacial.

$\mathrm{O}$ alcance (a) estabelece o limite de dependência espacial entre as amostras, isto é, para distâncias iguais ou menores que o alcance, pode-se dizer que os valores vizinhos de uma variável estão espacialmente correlacionados e podem ser utilizados para estimar valores em qualquer ponto entre eles (Reichardt, 1985). O patamar $(\mathrm{Co}+\mathrm{C} 1)$ é atingido quando a variância espacial dos dados (C1) se torna constante com o aumento da distância entre as amostras. Na Tabela 2 observa-se que os valores de alcance obtidos para as variáveis estudadas variaram de 20 a $40 \mathrm{~m}$, respectivamente.

Os solos afetados por sais são classificados em função dos valores do pHps, CEes e PST (Raij, 1991). Neste contexto e para fins de caracterização da salinidade na área estudada, foram tomados como base os valores medidos dessas variáveis e a classificação de solos apresentada por Richards (1954), seguindo-se metodologia adotada por Coelho \& Ferreyra (1986). Foram confeccionados mapas de isolinhas da combinação da CEes e PST utilizando-se o processo de interpolação inverso do quadrado da distância, uma vez que não é possível utilizar a krigagem na interpolação dos dados que não apresentam estrutura de dependência espacial. Pela Figura 4 pode-se comprovar o aumento dos problemas de salinidade com a profundidade no lado esquerdo da área, o que é evidenciado pela escala de tonalidades apresentada. Verifica-se que cerca de 24, 27 e 33\% da área estudada estão afetados por sais, nas profundidades de 0 - 20, 20 - 40 e 40 - 60 $\mathrm{cm}$, respectivamente. Resultados semelhantes foram obtidos por Coelho \& Ferreyra (1986) em solo aluvial no Perímetro Irrigado de Morada Nova, CE.

Por conseguinte, a área estudada foi dividida em duas subáreas: uma afetada pela salinidade $\mathrm{AF}$ atingindo cerca de $35 \%$ da área, e outra normal $(\mathrm{N})$, conforme Figura 4 tendo-se em vista o manejo do solo. Quanto à salinidade, supõe-se que não há restrição para o plantio de diversas culturas na sub-área N. Para a sub-área AF, sugerem-se estudos de variáveis físico-hídricas do solo (condutividade hidráulica, altura do lençol freático, classe textural, porosidade drenável) que permitam a adoção de critérios visando à construção de drenos subterrâneos, coletores e interceptores (Millar, 1988) de modo que o excesso de água proveniente da chuva ou irrigação possa ser removido; depois, pode-se fazer a aplicação de corretivos adequados, tomando-se como base os valores da PST e CTC (Cruciani, 1987) seguida da recuperação da área, mediante lavagem do excesso de sais solúveis. Culturas tolerantes à salinidade (por exemplo: arroz, algodão e algaroba) podem ser cultivadas no local. As lâminas de irrigação devem ser aplicadas de modo a manter um balanço favorável de sais no solo e evitar a elevação do lençol freático.

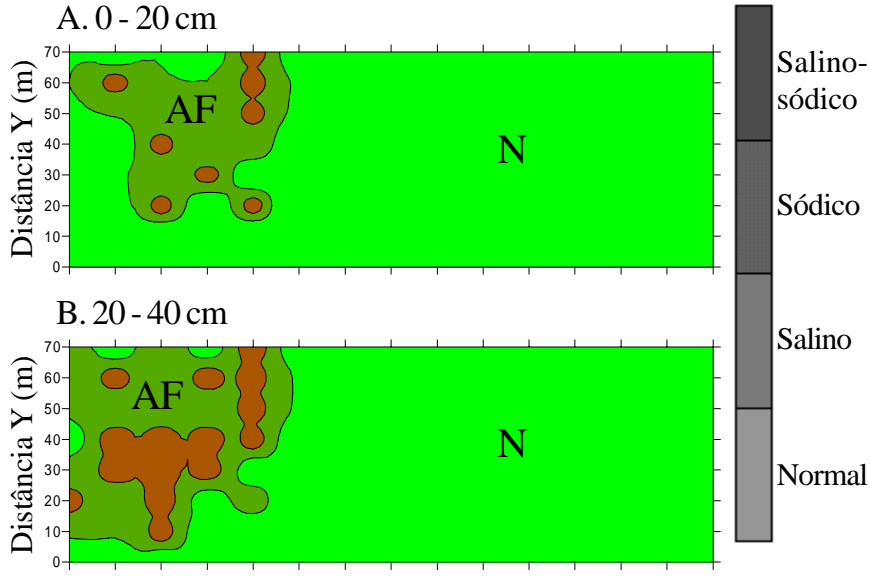

C. $40-60 \mathrm{~cm}$

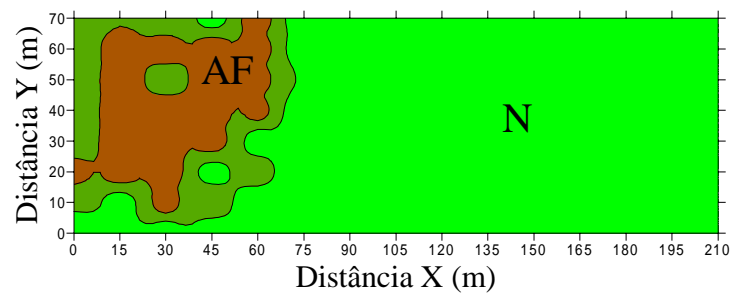

Figura 4. Mapas de classes de solo salinizado para as amostragens de $0-20 \mathrm{~cm}$ (A), $20-40 \mathrm{~cm}$ (B) e $40-60 \mathrm{~cm}$ (C) evidenciando as sub-áreas afetadas pela salinidade (AF) e $\operatorname{normal}(\mathrm{N})$

Como forma de se impedir o aumento do problema de sais, a sub-área afetada deve ser isolada da normal, através da construção de diques (Pizarro, 1978; Medeiros \& Gheyi, 1997). Na realização de pesquisas, procedimentos diferenciados devem ser adotados levando-se em consideração a subdivisão da área e sua variabilidade espacial.

\section{CONCLUSÕES}

1. Das variáveis estudadas, apenas o pHps apresentou distribuição normal, em todas as profundidades estudadas. Foi observada baixa variabilidade para o $\mathrm{pH}(\mathrm{CV}$ de 8,25 a 10,91\%) e elevada para a CEes (CV entre 135,85 e 173,15\%) e PST (CV de $157,95$ a $172,61 \%)$.

2. Aos semivariogramas experimentais que apresentaram estrutura de dependência espacial de dados, foram ajustados modelos esférico e gaussiano, com alcances variando de 20 a $40 \mathrm{~m}$. Com o exame dos semivariogramas foi detectada de fraca a forte dependência espacial.

3. Os mapas de isolinhas obtidos da combinação das CEes e PST mostraram que os problemas de salinidade e sodicidade aumentaram com a profundidade.

\section{REFERÊNCIAS BIBLIOGRÁFICAS}

ALBUQUERQUE, J.A.; FIORIN, J.E.; REINERT, D.J.; MUTTI, L.S.M.; SILVA, E.P. da. Variabilidade espacial do solo e produção do milho. Revista Brasileira de Ciência do solo, Campinas, v.20, n.1, p.151-157, 1996.

BECKETTI, P.H.T.; WEBSTER, R. Soil variability: A review. Soils and Fertilizers, Ferns, v.34, p.1-15, 1971. 
BRASIL/SUDENE. I. Levantamento exploratório Reconhecimento dos solos da Paraíba. II. Interpretação para uso agrícola dos solos da Paraíba. Convênio de mapeamento de solos. Rio de Janeiro: MA/EPE/USAID/BRASIL. Série Pedológica n. 8, 683p. 1972. Boletim Técnico, 15.

CAMBARDELLA, C.A.; MOORMAN, T.B.; NOVAK, J.M.; PARKIN, T.B.; KARLEM, D.L.; TURCO, R.F.; KONOPA, A.E. Field scale variability of soil properties in central Iowa soil. Soil Science Society of America Journal, Madison, v.58, p.1501-1511, 1994.

CARVALHO, O.S. Variabilidad espacial y temporal de la materia organica en un typic xerofluvent sometido a distintas sucessiones del cultivo. Madrid, Escuela Tecnica Superior de Ingenieros Agronomia. Universidad Politécnica de Madrid. 1996. 146p. Tese Doutorado.

COELHO, M.A.; FERREYRA, F.F. Characterization of saltaffected soil in the semi-arid region of Northeastern Brazil. Turrialba, São José, v.36, n.2, p.171-178, 1986.

COSTA NETO, P.L.O. Estatística. 15ed. São Paulo: Edgard Blucher, 1997.264p.

CRUCIANI, D.E. A drenagem na agricultura. 4ed. São Paulo: Nobel, 1987.337p.

EMBRAPA. Centro Nacional de Pesquisa de Solo. Manual de métodos de análise de solo. 2 ed. Rio de Janeiro: EMBRAPA. Serviço Nacional de Levantamento e Conservação de Solo, 1997.212 p.

FAO/UNESCO - Irrigation, drainage and salinity. An international sourcebook. Paris. HUTCHINSON/FAO/UNESCO, 1973. 510p.

GUERRA, P.A.G. Geoestatística operacional. Brasília: Ministério das Minas e Energia, Departamento de Produção Mineral, 1988. 145p.

HOAGLIN, D.C.; MOSTELLER, F.; TYKEY, J.W. Análisis exploratória de datos: Técnicas robustas, un guia. Lisboa: Salamandra, 1983.446p.
MEDEIROS, J.F. de; GHEYI, H.R. Manejo do sistema solo-águaplanta em solos afetados por sais. In: GHEYI, H.R.; QUEIROZ, J.E.; MEDEIROS, J.M. (ed.) Manejo e controle da salinidade na agricultura irrigada. Campina Grande: UFPB/SBEA, 1997. p. 239-288.

MILLAR, A. A drenagem de terras agrícolas: Bases agronômicas. São Paulo: Editerra Ltda., 1988. 306p.

OLIVEIRA, J.J. de. Variabilidade de características químicas de um solo salino-sódico. Campina Grande: Centro de Ciências e Tecnologia da Universidade Federal da Paraíba. 1998. 101p. Dissertação Mestrado.

OLIVEIRA, M. de. Gênese, classificação e extensão de solos afetados por sais. In: GHEYI, H.R.; QUEIROZ, J.E.; MEDEIROS, J.M. (ed.) Manejo e controle da salinidade na agricultura irrigada. Campina Grande: UFPB/SBEA, 1997. p.1-37.

PARAÍBA, GOVERNO DO ESTADO - Superintendência de Obras do Plano de Desenvolvimento do Estado. Projeto de Irrigação Capoeira: Estudo de viabilidade (Tomo I Diagnóstico), 1989.53p.

PIZARRO, F. Drenaje agrícola y recuperacion de suelos salinos. Madrid: Agrícola Española, 1978. 528p.

RAIJ, B.V. Fertilidade do solo e adubação. Piracicaba: Agronômica Ceres, 1991.343p.

REICHARDT, K. Variabilidade espacial e temporal de solos. In: Processos de transferência no sistema solo-planta-atmosfera. 4ed. Campinas: Fundação Cargill, 391 - 416p. 1985.

RICHARDS, L.A. Diagnosis and improvement of saline and alkali soils. Washington, D.C.: United States Salinity Laboratory, 1954. 160p. United States Department of Agriculture Handbook, 60

WARRICK, A.W.; NIELSEN, D.R. Spatial variability of soil physical properties in the field. In: HILLEL, D. (ed.) Applications of soil physics. New York: Academic Press, 1980. p.319-344. 